\title{
GP6 promotes the development of cerebral ischemic stroke induced by atherosclerosis via the FYN-PKA-pPTK2/FAK1 signaling pathway
}

\author{
Ye Gu ${ }^{1, A-C, F}$, Yihua Wu $\mathrm{Wu}^{1, B, C, F}$, Liang Chen $^{2, A, D-F}$ \\ ${ }^{1}$ Shanghai International Medical Center, China \\ ${ }^{2}$ Xinhua Hospital affiliated to Shanghai Jiaotong University School of Medicine, China \\ A - research concept and design; $\mathrm{B}$ - collection and/or assembly of data; $\mathrm{C}$ - data analysis and interpretation; \\ $D$ - writing the article; $E$ - critical revision of the article; $F$ - final approval of the article
}

\section{Address for correspondence \\ Liang Chen}

E-mail:I_chen15@21cn.com

Funding sources

None declared

Conflict of interest

None declared

Received on November 23, 2020

Reviewed on January 6, 2021

Accepted on April 6, 2021

Published online on August 20, 2021

Cite as

Gu Y, Wu Y, Chen L. GPG promotes the development of cerebral ischemic stroke induced by atherosclerosis via the FYN-PKA-PPTK2/FAK1 signaling pathway. Adv Clin Exp Med. 2021;30(8):823-829.

doi:10.17219/acem/135510

DOI

10.17219/acem/135510

Copyright

Copyright by Author(s)

This is an article distributed under the terms of the

Creative Commons Attribution 3.0 Unported (CC BY 3.0)

(https://creativecommons.org/licenses/by/3.0/)

\begin{abstract}
Background. Cerebrovascular disease is currently a serious threat to human health and life, commonly including cerebral infarction, cerebral hemorrhage and transient cerebral ischemia, among others.

Objectives. To explore the role and e molecular mechanism of GP6 in the development of cerebral ischemic stroke (CIS) induced by atherosclerosis (AS).

Materials and methods. Forty-five male New Zealand white rabbits were randomly divided into 3 groups: the control, CIS model and anti-GP6 group. Carotid artery tissues and blood of the white rabbits were collected for analysis. Hematoxylin and eosin (H\&E) staining was used to analyze the pathological characteristics of vascular endothelial injury. Flow cytometry (FCM) was performed to analyze the content of Th1 and Th17 in blood. Immunohistochemistry was used to analyze the distribution and relative expression of FCER1G, ITGA2 and GP6 proteins in the carotid artery and cerebrovascular tissues. Western blot was applied to determine the protein expression of GP6, FYN, PKA, PPTK2, and PFAK1 in carotid artery tissues of the rabbits.
\end{abstract}

Results. In the CIS model group, there was lymphocyte infiltration, fibrous tissue formation, and the formation of thrombus and lipid plaques. In the anti-GP6 group, scattered thin plaques were observed, and no obvious foam cell deposition was observed. The Th1 and Th17 content was significantly decreased in the CIS model group compared to the control and anti-GP6 group. The relative expression of FCER1G, ITGA2 and GP6 in the CIS model group was significantly higher compared to those in the control group and anti-GP6 group. The protein expression of GP6, FYN, PKA, pPTK2, and PFAK1 in the CIS model group were markedly higher compared to those in the control group and anti-GP6 group.

Conclusions. GP6 can promote the development of CIS by activating the FYN-PKA-PPTK2/FAK1 signaling pathway.

Key words: CIS, AS, GP6, thrombus, vascular endothelial cells 


\section{Background}

Cerebrovascular disease is currently a serious threat to human health and life, the most common being cerebral infarction, cerebral hemorrhage and transient cerebral ischemia, among others. ${ }^{1}$ It mainly occurs in middle-aged and elderly people, but the age of onset has shown a trend of decreasing, often occurring in people $30-50$ years old. ${ }^{2}$ The disease often leads to cognitive disorders or disorders affecting the physical activity of patients. Many patients are unable to take care of themselves after onset and quality of life is seriously reduced, resulting in them becoming a heavy burden to their families and society. ${ }^{3}$ At present, there is no radically effective cure for the disease, and the prognosis is often closely related to the initial severity of the disease. ${ }^{4}$ Even if patients survive, most of them are accompanied by lifelong sequelae, and cerebrovascular diseases can occur repeatedly, causing far more pain to the patients and their families than malignant tumors and cardiovascular diseases. Therefore, how to prevent and treat cerebrovascular disease more effectively is the most serious problem that neurology faces presently. ${ }^{5}$ Cerebral ischemic stroke (CIS) is ischemic necrosis or softening of localized brain tissue due to cerebral blood circulation disorder, ischemia or hypoxia. It is the most common type of cerebrovascular disease, accounting for about $70 \%$ of all acute cerebrovascular diseases. About 30-40\% of CIS is caused by carotid atherosclerotic stenosis. ${ }^{6}$

Extracellular matrix (ECM) contact exposed at the site of platelet and vascular injury is the first line of defense for repairing damaged tissue and stopping bleeding. Collagen, one of the macromolecular components of ECM, can not only adhere to platelets through direct and indirect channels but also cause platelet aggregation and expression of pro-coagulant activity. The interaction between platelets and collagen in arteries or damaged vessels with high shear stress is particularly important. Current research suggests that platelets have 2 types of collagen receptors on their surfaces, $\alpha 2 \beta 1$ and glycoprotein 6 (GP6). It is a glycoprotein receptor that acts on collagen and is located on the long arm of chromosome 19 (19q13.4). It contains 8 exons and has a molecular weight of $62 \mathrm{kDa}$. In humans, GP6 is encoded by the GP6 gene. GP6 mediates the initial adhesion of platelets to collagen, generates signal transduction, improves the binding affinity of integrin receptors, and causes platelet aggregation, platelet release and thrombosis. ${ }^{7}$ When serum GP6 concentration is increased, it can promote the initial adhesion of collagen and platelets, improving the affinity of the integrin receptor, which induces platelet aggregation and thrombosis. ${ }^{8}$ Kubisz et al. found that a variation of the GP6 gene was related to platelet aggregation. ${ }^{9}$ Sokol et al. found that a GP6 gene polymorphism may be related to the heightened aggregation ability of platelets..$^{10}$ Despite the complex composition of the ECM, the adhesion of platelets and collagen plays an important role in initiating hemostasis and thrombus in the body. Inhibition of GP6 function can significantly inhibit collagen-induced platelet adhesion, aggregation and platelet thrombosis under high shear stress in vitro. ${ }^{11}$ Therefore, we believe that atherosclerotic CIS is closely related to the structural and functional integrity of GP6, but the mechanism of action of GP6 in atherosclerosis (AS) has rarely been studied.

\section{Objectives}

In this paper, we wanted to investigate the mechanism of action of GP6 in AS. Helper T (Th) cell content, Th1 and Th17, was significantly decreased in the CIS model group compared to the control group, while having no marked differences compared to the anti-GP6 group. Immunohistochemistry showed that the proteins FCER1G, ITGA2 and GP6 were all distributed in the cell membrane. GP6 can promote the formation of cerebral ischemic stroke via upregulating the expression of FCER1G, ITGA2, FYN, PKA, $p P T K 2$, and $p F A K 1$. This may be a new target for treating cerebral ischemic stroke.

\section{Materials and methods}

\section{Model establishment and grouping}

Forty-five male New Zealand white rabbits ( 1618 weeks old, weight: $\sim 1.5-2.0 \mathrm{~kg}$ ) were obtained from the Naval Medical Institute Animal Center (Shanghai, China). They were randomly divided into 3 groups ( $\mathrm{n}=15$ in each group): the control group, CIS group and anti-GP6 group. In the CIS group and the anti-GP6 group, rabbits were first fed with a specific high-fat diet for 8 weeks, and then $3 \%$ hydrogen peroxide solution was applied to perfuse the bilateral common carotid arteries of the rabbits, causing vascular endothelial oxidative stress injury. In the antiGP6 group, the rabbits were given anti-GP6 antibody via the tail vein. Carotid artery tissues of the white rabbits were collected and rapidly stored in $-80^{\circ} \mathrm{C}$ liquid nitrogen for further use.

In this study, atherosclerotic stenosis injury rabbit models were constructed and separated into 3 groups: the control group, CIS group and anti-GP6 group. Hematoxylin and eosin (H\&E) staining was used to analyze the pathological features of vascular endothelial injury. Flow cytometry (FCM) was used to determine the concentration of Th1 and Th17 cells in blood samples. The expressions of GP6, FYN, PKA, pPTK2, and FAK1 were also assessed.

The animal experiments were approved by the Ethics Committee of Xinhua Hospital affiliated with Shanghai Jiaotong University School of Medicine (Shanghai, China). 


\section{Hematoxylin and eosin staining}

After the rabbits were sacrificed by the cervical dislocation method, hippocampal tissue was taken from their brains. The tissues were fixed with $4 \%$ formaldehyde for $24 \mathrm{~h}$, embedded in paraffin and sectioned into $5 \mu \mathrm{m}$-thick sections. Histopathological changes were observed under a light microscope (Olympus Corp., Tokyo, Japan) after H\&E staining.

\section{Flow cytometry}

Cells were digested with trypsin, washed twice with phosphate-buffered saline (PBS) and collected into a centrifuge tube. Binding buffer was added to prepare cell suspensions with a final concentration of $1 \times 10^{6} / \mathrm{mL}$. Annexin V-FITC kit (BioVision, Milpitas, USA) instructions were used for labeling, which was performed according to the instructions of Annexin V-FITC kit. Annexin $\mathrm{V}$ was added for staining at room temperature in the dark for $15 \mathrm{~min}$.

\section{Western blot}

Cells were lysed with cell lysis buffer on ice for $30 \mathrm{~min}$ and then centrifuged at 12,000 rpm for $25 \mathrm{~min}$. Supernatant was collected and the Bradford method was used for protein quantification. Protein in the amount of $25 \mu \mathrm{g}$ was loaded for sodium dodecyl sulphate-polyacrylamide gel electrophoresis (SDS-PAGE). The proteins were transferred to a polyvinylidene difluoride (PVDF) membrane and sealed with $5 \%$ skim milk at room temperature for $1 \mathrm{~h}$; then, primary antibody was added and the membrane was incubated overnight at $4^{\circ} \mathrm{C}$. The next day, the secondary antibody was added and incubated at room temperature for $1 \mathrm{~h}$, and then, electrochemiluminescence (ECL) hypersensitive luminescence solution was added for developing.

\section{Immunohistochemistry}

Tissues were dewaxed and endogenous oxidase was inactivated with $3 \%$ hydrogen peroxide. After washing in PBS 3 times, the antigen was retrieved by microwave heating. The slices were placed in sodium citrate buffer with pH 6.0, heated to boiling in a microwave and then cooled. The slices were again microwaved in sodium citrate buffer with $\mathrm{pH} 6.0$ and then washed with PBS. After cleaning, sections were removed and sealed for $10 \mathrm{~min}$. Then, mouse anti-human FCER1G, ITGA2 and GP6 monoclonal antibodies $(1: 150)$ were added and incubated at $4^{\circ} \mathrm{C}$ for $12 \mathrm{~h}$. After washing, biotin-labeled secondary antibodies were added and incubated at $4^{\circ} \mathrm{C}$ for $20 \mathrm{~min}$ each. After incubation and washing, the samples were washed again and immersed in DAB color developing solution for $5 \mathrm{~min}$. Then, the samples were washed, re-dyed, dehydrated, sealed and observed under an optical microscope.

\section{Statistical analyses}

IBM SPSS v. 19.0 software (IBM Corp., Armonk, USA) was used for statistical analysis. The results were expressed as mean values \pm standard deviation (SD). Differences between groups were compared using one-way analysis of variance (ANOVA) tests followed by Bonferroni post hoc test. The assumption for ANOVA was verified with the Brown-Forsythe test. A value of $\mathrm{p}<0.05$ was considered statistically significant.

\section{Results}

\section{Anti-GP6 inhibits vascular endothelial injury}

After the successful establishment of the evaluation models, the rabbits were anesthetized with isopentane and sacrificed. Carotid artery tissue and cerebrovascular tissues were collected from the white rabbits, and $H \& E$ staining was used to analyze the pathological characteristics of the vascular endothelial injury. As shown in Fig. 1, in the control group, the smooth muscle cells were arranged neatly, tightly and orderly, the endothelial layer was continuous and there was no lipid deposition in the subcutaneous tissue. The structure of each layer was clear. In the CIS group, the endothelium was discontinuous, the intimal hyperplasia was serious, the tube wall was full of plaques, a large number of foam cells could be seen, the membranes in the middle and outside were irregularly thickened, and foam cell infiltration could be seen. In the anti-GP6 group, endothelial cells were continuous, lipid deposition was observed under the intima, scattered thin plaques were observed, and no obvious foam cell deposition was observed. The thickness of the membrane was the same as that of the control group membrane, which was thinner than the CIS model group membrane.

\section{Content of Th1 and Th17}

The content of Th1 and Th17 in blood was analyzed with FCM and the results are shown in Fig. 2. The Th1 $(\mathrm{p}<0.01$, degrees of freedom $(\mathrm{df})=2, \mathrm{~F}=337.7)$ and Th17 ( $\mathrm{p}<0.01, \mathrm{df}=2, \mathrm{~F}=150.6)$ content was significantly decreased in the CIS model group compared to the control group, while there was no marked difference compared to the anti-GP6 group.

\section{Results of immunohistochemistry}

Immunohistochemistry was used to analyze the distribution and relative expression of FCER1G, ITGA2 and GP6 proteins in the carotid artery and cerebrovascular tissues of white rabbits. The proteins FCER1G, ITGA2 and GP6 were all distributed in the cell membrane 

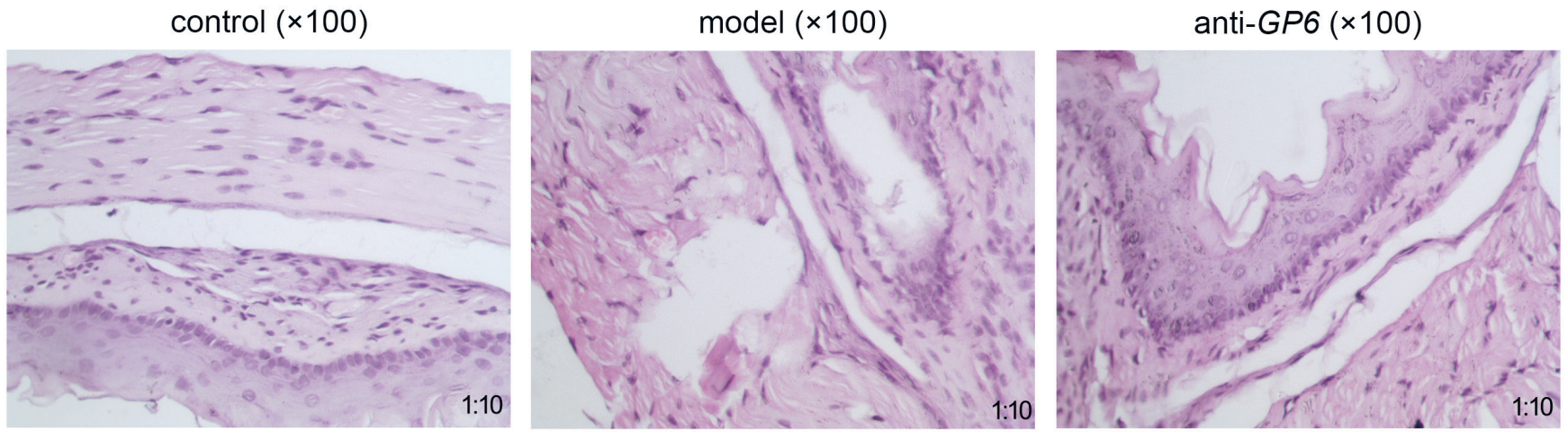

Fig. 1. The pathological features of vascular endothelial injury in the control, cerebral ischemic stroke (CIS) model and anti-GP6 groups analyzed using H\&E staining. In the control group, the smooth muscle cells were arranged neatly, tightly and orderly, the endothelial layer was continuous and there was no lipid deposition in the subcutaneous tissue; in the CIS model group, the endothelium was discontinuous, the intimal hyperplasia was serious, the tube wall was full of plaques, and a large number of foam cells could be seen; and in the anti-GP6 group, scattered thin plaques were observed and no obvious foam cell deposition was observed
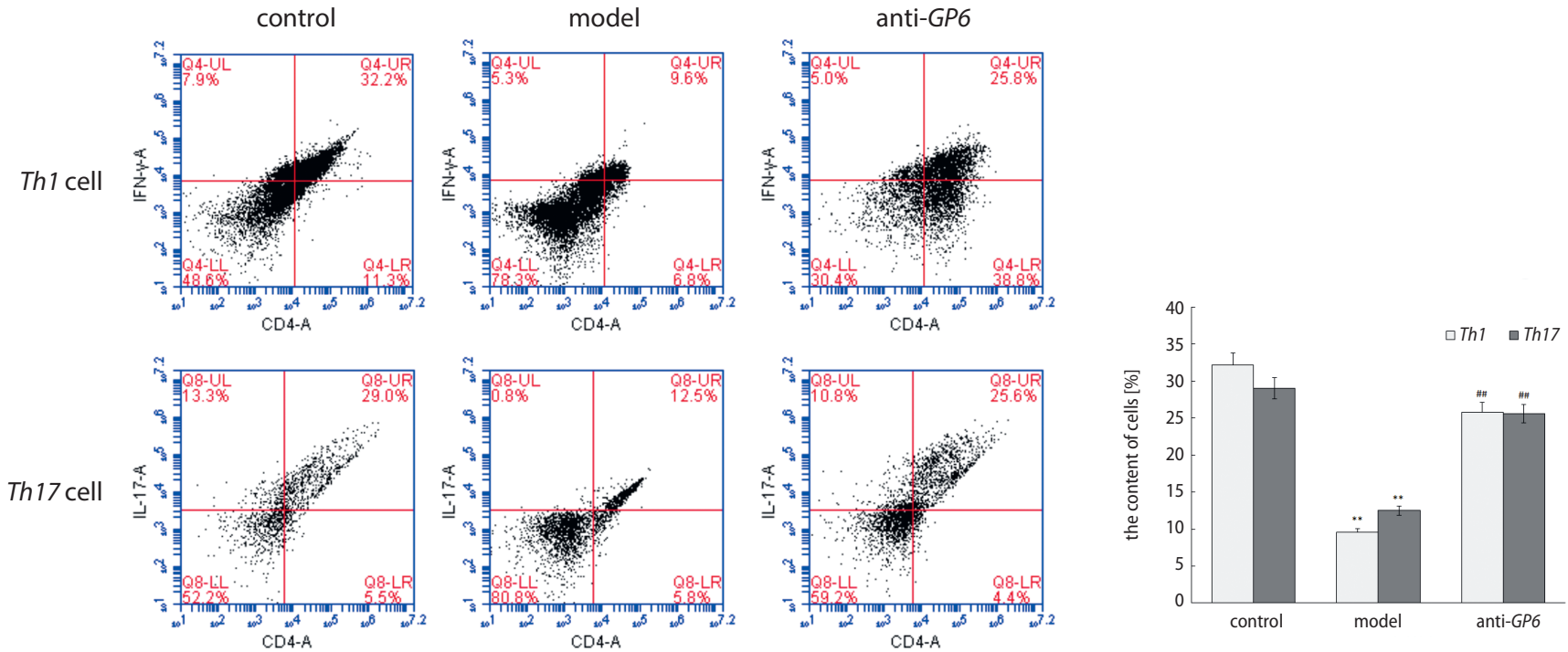

Fig. 2. The content of Th1 and Th17 in blood samples of rabbits from the control, cerebral ischemic stroke (CIS) model and anti-GP6 groups determined with flow cytometry (FCM). The Th1 and Th17 content was significantly decreased in the CIS model compared to the control group and anti-GP6 group

${ }^{*} \mathrm{p}<0.05$ or ${ }^{\# \#} \mathrm{p}<0.01$, indicating the significant difference using one-way analysis of variance (ANOVA) followed by Bonferroni post hoc test.

(Fig. 3). The relative expression of FCER1G, ITGA2 and GP6 in the CIS model group was significantly higher compared to the control group and anti-GP6 group. The relative expression of FCER1G, ITGA2 and GP6 did not significantly differ between the control group and anti-GP6 group.

\section{Expression of GP6, FYN, PKA, pPTK2, and $P F A K 1$}

Western blot was applied to determine the protein expression of GP6, FYN, PKA, $p P T K 2$, and $p F A K 1$ in carotid artery tissues of rabbits from the control, CIS model and anti-GP6 group. The protein expression of GP6 (p $<0.001$, $\mathrm{df}=2, \mathrm{~F}=392.9), F Y N(\mathrm{p}<0.001, \mathrm{df}=2, \mathrm{~F}=380.3), P K A$ $(\mathrm{p}<0.001, \mathrm{df}=2, \mathrm{~F}=304.3), p P T K 2(\mathrm{p}<0.001, \mathrm{df}=2$, $\mathrm{F}=369.9)$, and $p F A K 1(\mathrm{p}<0.001, \mathrm{df}=2, \mathrm{~F}=562.8)$ in the CIS model group were markedly higher compared to those in the control group and anti-GP6 group (Fig. 4). Moreover, the expression of GP6, FYN, PKA, pPTK2, and $p F A K 1$ were significantly upregulated in the anti-GP6 group compared with the control group.

\section{Discussion}

With a remarkable rise in people's living standards and an accelerated pace of life, the onset of CIS is observed earlier than before and the incidence rate increases year after year. In particular, progressive CIS has a poor prognosis and high morbidity and mortality, which poses a serious threat to people's health and life safety, and burdens patients and families. ${ }^{12,13}$ Carotid AS is an important risk factor for CIS and is closely related to the occurrence, development and recurrence of cerebral infarction. ${ }^{14}$ To date, surgery 


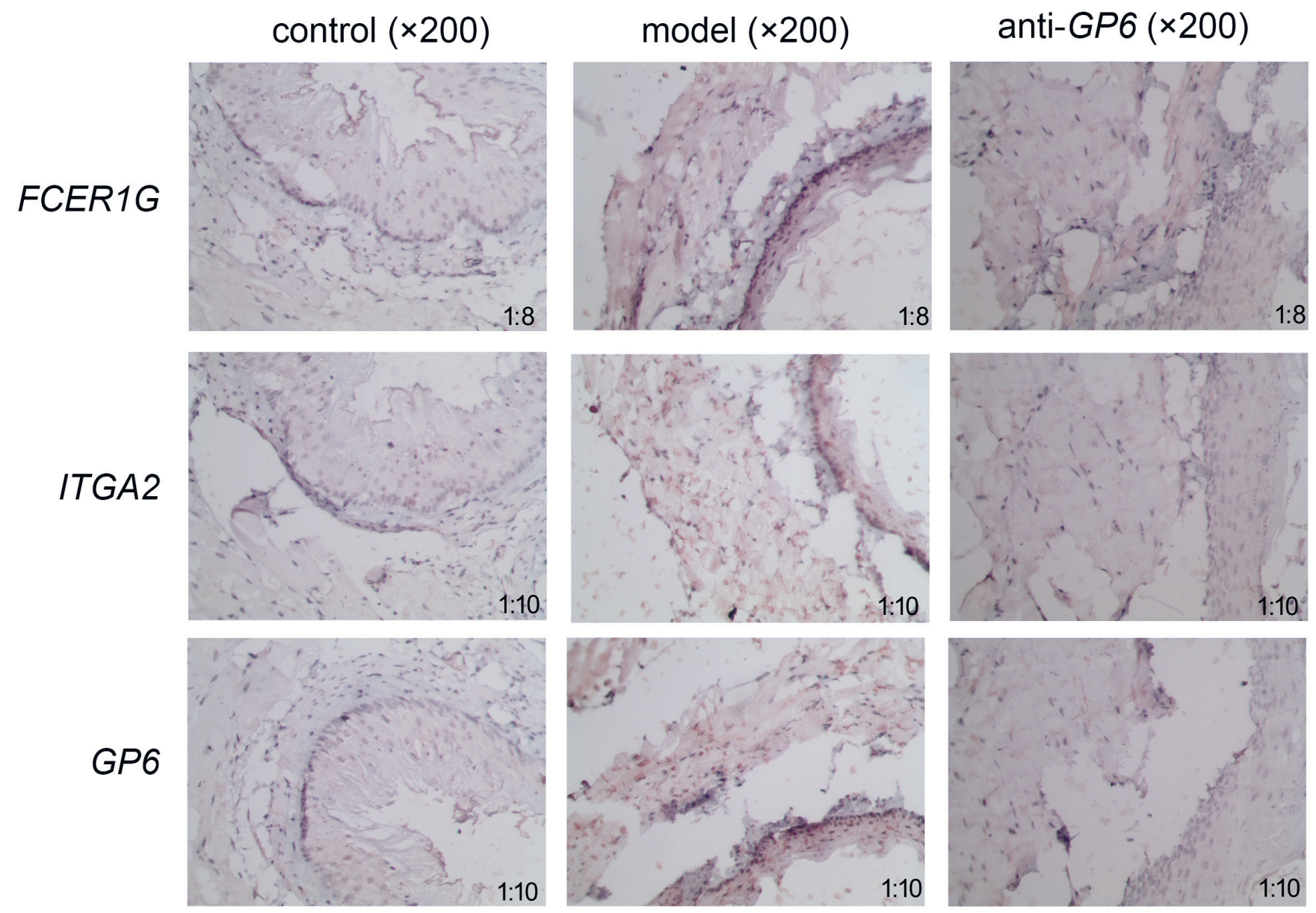

Fig. 3. Immunofluorescent staining of FCER1G, ITGA2 and GP6 proteins in the carotid artery and cerebrovascular tissues of white rabbits in the control, cerebral ischemic stroke (CIS) model and anti-GP6 groups. The proteins FCER1G, ITGA2 and GP6 were all distributed in the cell membrane. The relative expression of FCER1G, ITGA2 and GP6 in the CIS model group was significantly higher compared to the control group and anti-GP6 group

A

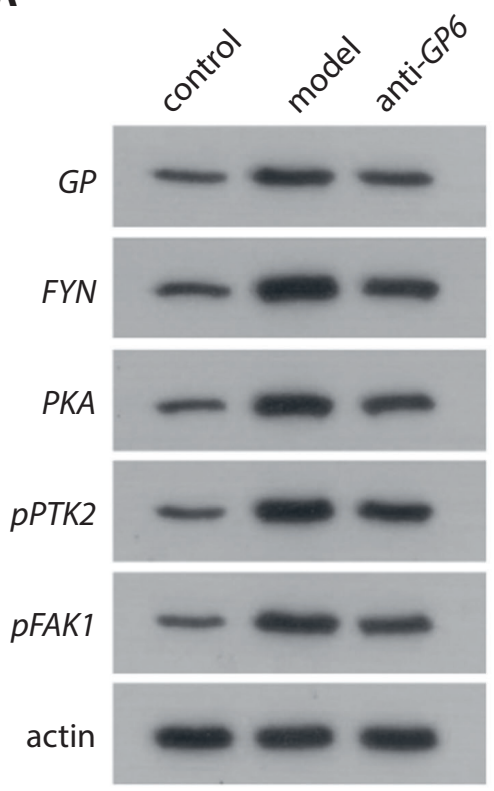

B

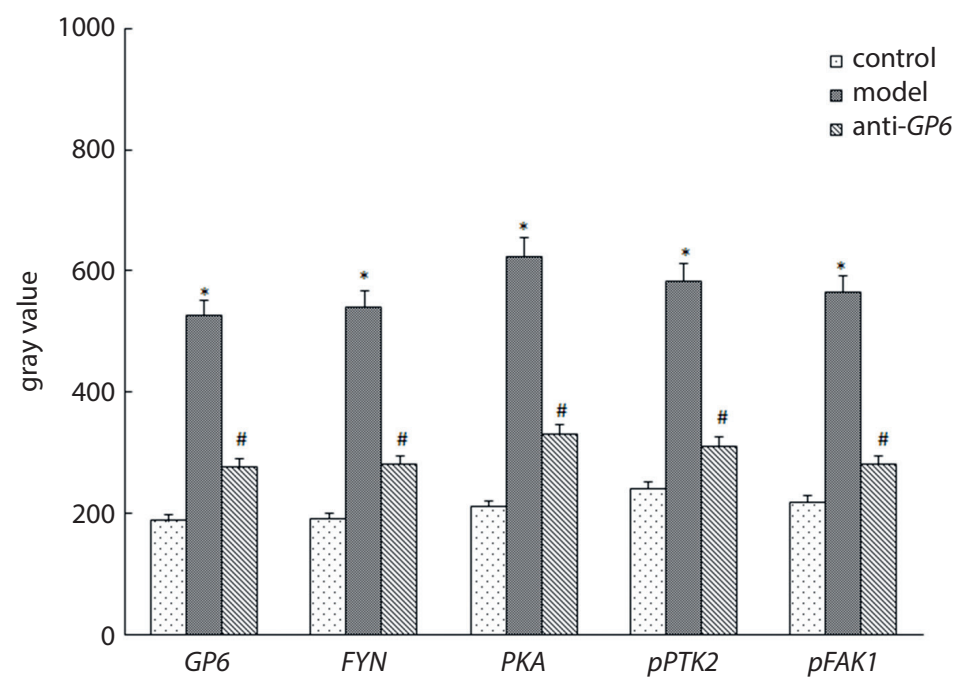

Fig. 4. The protein expression of GP6, FYN, PKA, PPTK2, and PFAK1 in carotid artery tissues of rabbits in the control, cerebral ischemic stroke (CIS) model and anti-GP6 groups measured with western blot assay. The protein expression of GP6, FYN, PKA, pPTK2, and pFAK1 in the CIS model group was markedly higher compared to the control group and anti-GP6 group

${ }^{*} p<0.001$ or ${ }^{\#} p<0.01$, indicating the significant difference using one-way analysis of variance (ANOVA) followed by Bonferroni post hoc test. 
or endovascular treatment for patients with recent ischemic diseases has been dependent on the degree of narrowing of the responsible vessel. For many years, the extent of artery stenosis has been regarded as the most reliable index to predict the risk of stroke in patients with AS. Thromboembolism derived from atherosclerotic plaque is considered to be the main pathogenesis of CIS in most atherosclerotic patients. Plaque rupture can lead to platelet aggregation, local thrombosis or thromboembolism. Plaque itself can also cause thromboembolism. ${ }^{15}$ Protein function analysis revealed that GP6 is a platelet glycoprotein, which plays an important role in platelet coagulation, thrombosis and arterial embolization. ${ }^{16}$ The GP6 signaling pathway mainly activates FYN and $P K A$, phosphorylates PTK2/FAK1 and promotes platelet adhesion to damaged vascular endothelium.

Vascular endothelial cells have anticoagulant and fibrinolytic properties and can influence the chemotaxis and adhesion properties of cell membranes. ${ }^{17}$ Under normal circumstances, vascular endothelial cells cover the inner surface of the entire vascular system, providing an anticoagulant interface that prevents platelets and other blood cells from adhering to the subcutaneous tissue and preventing activation of the clotting response. Endocrine dysfunction caused by endothelial cell injury plays a key role in the pathophysiological mechanism of the early formation and development of AS. ${ }^{18}$ In this study, male New Zealand white rabbits were randomly divided into 3 groups: control, CIS model and anti-GP6 group. Results of $H \& E$ staining analysis of the vascular endothelial injury showed that in the CIS model group, the endothelium was discontinuous, the intimal hyperplasia was serious, the tube wall was full of plaques, a large number of foam cells could be seen, the membranes in the middle and outside were irregularly thickened, and foam cell infiltration could be seen. However, the injury degree was reduced in the anti-GP6 group compared to the CIS model group. Results of FCM showed that Th1 and Th17 content was significantly decreased in the CIS model group compared to the control group, while it did not markedly differ from the anti-GP6 group. Atherosclerosis occurs for various reasons, including the involvement of macrophages, $\mathrm{T}$ lymphocytes, endothelial cells, smooth muscle cells, mast cells, and other cells. After entering the vascular wall, T lymphocytes are activated under antigen stimulation to produce inflammatory cytokines, which can aggravate the progression of AS by amplifying the inflammatory response. Different $T$ cell subsets in the vascular wall are not only involved in the early plaque formation of AS but also promote the progression of AS, which is closely related to the pathological process of AS. ${ }^{19}$ The CD $4{ }^{+} \mathrm{T}$ cells play a crucial role in the development of AS. According to biological characteristics and different cytokines produced, CD4 ${ }^{+} \mathrm{T}$ cells are mainly divided into Th1, Th2 and Th17. The Th1 cells are differentiated from initial $T$ cells in response to interleukin (IL)-12 and produce interferon gamma (IFN- $\gamma$ ), which acts as a defense against microorganisms in the cell.
The role of Th17 cells in disease is mainly to promote defense against organ-specific autoimmune diseases and chronic infections. Results of animal experiments carried out by Davenport et al. showed that Th1 and Th2 immune responses were involved in the formation of atherosclerotic lesions. ${ }^{20} \mathrm{Kim}$ et al. assessed 124 patients with chest pain who underwent coronary angiography and found the expression of Th1 and Th17 cells in patients with stable angina pectoris was significantly increased, which indicated that Th1 and Th17 cells are associated with the development of AS. ${ }^{21}$ Interleukin 17 (IL-17) is a major effector of Th17 cells and an early promoter of T-cell-induced inflammatory response, which can amplify the inflammatory response. Immunohistochemistry results in our study showed the proteins FCER1G, ITGA2 and GP6 were all distributed in the cell membrane. The relative expression of FCER1G, ITGA2 and GP6 in the CIS model group was significantly higher compared to the control group and anti-GP6 group. The FCER1G gene is located on chromosome 1q23 (1:161185024-161190489) 24 and encodes an 86 -amino acid protein. ${ }^{22}$ ITGA2 is a protein-coding gene. Diseases associated with ITGA2 include anus disease and thrombocytopenia. ${ }^{23}$ Results from western blotting indicated that the protein expression of GP6, FYN, PKA, $p P T K 2$, and $p F A K 1$ in the CIS model group was markedly higher compared to the control group and anti-GP6 group. Protein kinases are enzymes that catalyze protein phosphorylation, which include an important protein kinase, protein kinase A $(P K A)$.

\section{Limitations}

At present, a large number of studies have been carried out on the GP6 gene in China and abroad. The consistent view is that GP6 gene polymorphisms are related to platelet aggregation ability, but whether they lead to an increase or decrease in platelet aggregation, is still in question. If a polymorphism leads to an increase in platelet aggregation, then it is likely to be associated with thrombotic diseases. However, some scholars found that the ti3254c gene polymorphism of GP6 is not significantly associated with the occurrence of coronary heart disease. Scholars generally agree that the expression of GP6 is enhanced in patients with acute ischemic diseases such as stroke and myocardial infarction. However, regarding whether GP6 gene polymorphisms are related to AS-based diseases (such as coronary heart disease and cerebral infarction), current research results are mostly negative and more in-depth studies are needed for verification. The safety of long-term drug use and the role of drug immunity and cardiovascular events need to be further explored. Chronic inflammation, which is repeated over a long time, can also lead to an inability to cure the disease. The presence of other pathways in the mechanism of GP6 in the treatment of AS requires further study. 


\section{Conclusions}

GP6 can promote the formation of cerebral ischemic stroke by upregulating the expression of FCER1G, ITGA2, $F Y N, P K A, p P T K 2$, and $p F A K 1$. It may be a new treatment target for cerebral ischemic stroke.

\section{ORCID iDs}

Ye Gu (1) https://orcid.org/0000-0002-9936-8125

Yihua Wu (1) https://orcid.org/0000-0001-7119-1770

Liang Chen 구 https://orcid.org/0000-0003-1978-3212

\section{References}

1. Caprio FZ, Sorond FA. Cerebrovascular disease. Med Clin North Am. 2019;103(2):295-308. doi:10.1016/j.mcna.2018.10.001

2. Xiang $\mathrm{YH}$. Influencing factors of secondary early epileptic seizures in patients with acute cerebrovascular disease. Pract J Cardiac Cerebral Pneumal Vasc Dis. 2017;25(07):150-152.

3. Jibo LI, Jing LI, Liu WP. Analysis of clinical effect of nalmefene combined with Xingnaojing injection in the treatment of cerebrovascular disease with disturbance of consciousness. Xinjiang Medical Journal. 2018;48(12):1344-1345.

4. Celikbilek A, Ismailogullari S, Zararsiz G. Neutrophil to lymphocyte ratio predicts poor prognosis in ischemic cerebrovascular disease. J Clin Lab Anal. 2014;28(1):27-31. doi:10.1002/jcla.21639

5. Mutuberría LR, Capote RD. Benefits of therapeutic physical exercise in patients with sequelae of cerebrovascular disease. Pflege. 2012;21(3):172. doi:10.1024/1012-5302.21.3.172

6. Sibani S, Dipankar C, Arijit B, Mrinal Kanti G. Cerebral ischemic stroke: Cellular fate and therapeutic opportunities. Front Biosci (LandmarkEd). 2019:24:435-450. PMID:30468665

7. Naitoh K, Hosaka Y, Shirakawa K, Furusako S. Establishment of immunoassay for platelet-derived soluble glycoprotein $\mathrm{VI}$, a novel platelet marer. J Immunol Methods. 2015;418:52-60. doi:10.1016/j.jim.2015. 01.010

8. Best D, Senis YA, Jarvis GE, Eagleton HJ, Watson SP. GPVI levels in platelets: Relationship to platelet function at high shear. Blood. 2003; 102(8):2811-2818. doi:10.1182/blood-2003-01-0231

9. Kubisz P, Ivankova J, Skerenova M, Stasko J, Holly P. The prevalence of the platelet glycoprotein VI polymorphisms in patients with sticky platelet syndrome and ischemic stroke. Hematology. 2013;17(6): 355-362. doi:10.1179/1024533212Z.000000000142

10. Sokol J, Biringer K, Skerenova M, et al. Platelet aggregation abnormalities in patients with fetal losses: The GP6 gene polymorphism. Fertil Steril. 2012;98(5):1170-1174. doi:10.1016/j.fertnstert.2012.07.1108
11. Lecut C, Feeney LA, Kingsbury G, et al. Human platelet glycoprotein VI function is antagonized by monoclonal antibody-derived Fab fragments. J Thromb Haemost. 2010;1(12):2653-2662. doi:10.1111/j. 1538-7836.2003.00495.x

12. Chen J, Cui C, Yang X, et al. MiR-126 affects brain-heart interaction after cerebral ischemic stroke. Trans/ Stroke Res. 2017;8(4):374-385. doi:10.1007/s12975-017-0520-z

13. Gao XL, Chen LY, Sun LQ, Xiao-Rong LI, Zheng GQ. Clinical efficacy and safety of edaravone combined with butylphthalide in treatment of acute cerebral ischemic stroke. Chinese J Clin Pharmacol. 2015,31(16):1569-1571.

14. Yang $\mathrm{OH}$, Shen W, Jia XD, Ru JP, Du WZ. Correlation of ultrasound detection of carotid atherosclerosis plaque with ischemic cerebral stroke. J Hainan Medical University. 2016;22(015):153-155.

15. Fuster V, Badimon JJ, Chesebro JH, Fallon JT. Plaque rupture, thrombosis, and therapeutic implications. Haemostasis. 2010;26(Suppl 4): 269-284. doi:10.1159/000217308

16. Kotulicová $D$, Chudy $P$, Skereňová $M$, Ivanková J, Dobrotová $M$, Kubisz P. Variability of GP6 gene in patients with sticky platelet syndrome and deep venous thrombosis and/or pulmonary embolism. Blood Coagul Fibrinolysis. 2012;23(6):543-547. doi:10.1097/MBC.0b013 e328355a808

17. Wang SW, Deng LX, Chen HY, Su ZQ, Ye SL, Xu WY. MiR-124 affects the apoptosis of brain vascular endothelial cells and ROS production through regulating PI3K/AKT signaling pathway. Eur Rev Med PharmacolSci.2018;22(2):498-505. doi:10.26355/eurrev_201801_14201

18. Clemmons DR. Modifying IGF1 activity: an approach to treat endocrine disorders, atherosclerosis and cancer. Nat Rev Drug Discov. 2007; 6(10):821-833. doi:10.1038/nrd2359

19. Tse K, Tse H, Sidney J, Sette A, Ley K. T cells in atherosclerosis. Int Immunol. 2013(11):615-622. doi:10.1093/intimm/dxt043

20. Davenport $P$, Tipping PG. The role of interleukin-4 and interleukin-12 in the progression of atherosclerosis in apolipoprotein E-deficient mice. Am J Pathol. 2003;163(3):1117-1125. doi:10.1016/S00029440(10)63471-2

21. Kim JD, Lee SH, Seo EH, et al. Role of Th1 and Th17 cells in the development and complexity of coronary artery disease: comparison analysis by the methods of flow cytometry and SYNTAX score. Coron Artery Dis. 2015;26(7):604-611. doi:10.1097/MCA.0000000000 000289

22. Amo G, García-Menaya J, Campo P, et al. A nonsynonymous FCER1B SNP is associated with risk of developing allergic rhinitis and with IgE levels. Sci Rep. 2015;6(1):19724. doi:10.1038/srep19724

23. Felipe RLV, Tashi T, Koul P, Camp NJ, Prchal JT. Inherited giant platelet disorder, Kashmiri thrombocytopenia, a common syndrome found in Srinagar, India. Blood. 2014;124(21):4211. doi:10.1182/blood. V124.21.4211.4211 